\title{
OBESIDAD EN EL NIÑO: FACTORES DE RIESGO Y ESTRATEGIAS PARA SU PREVENCIÓN EN PERÚ
}

\author{
Carlos M. Del Águila Villar ${ }^{1,2, a}$
}

\begin{abstract}
RESUMEN
La prevalencia del sobrepeso y la obesidad en niños y adolescentes constituyen un problema de salud pública emergente en el Perú, por lo que es necesario conocer los diversos factores de riesgo para establecer medidas de prevención oportunas y eficientes, las cuales deben formar parte de las estrategias de salud, como propiciar la actividad física y una alimentación saludable, para asegurar que la población infantil llegue a la adultez sin enfermedades crónicas y con una adecuada calidad de vida.
\end{abstract}

Palabras clave: Obesidad; Factores de riesgo; Niño (fuente: DeCS BIREME).

\section{OBESITY IN CHILDREN: RISK FACTORS AND STRATEGIES FOR ITS PREVENTION IN PERU}

\begin{abstract}
The prevalence of overweight and obesity in children and adolescents represents an emerging public health problem in Peru, so it is necessary to be aware of the different risk factors in order to establish suitable and efficient prevention measures. These should contribute to health strategies such as promoting physical activity and a healthy diet to ensure that the infant population reaches adulthood without chronic diseases and with an adequate quality of life.
\end{abstract}

Key words: Obesity; Risk factors; Child (source: MeSH NLM).

\section{INTRODUCCIÓN}

La obesidad es una enfermedad sistémica, crónica y multifactorial en la que participan la susceptibilidad genética y los estilos de vida y del entorno, con influencia de diversos factores determinantes subyacentes, como la globalización, la cultura, la condición económica, la educación, la urbanización y los entornos político y social ${ }^{(1)}$.

La obesidad en el niño y adolescente es considerada un problema de salud pública tanto en los países desarrollados como en países en desarrollo, por el incremento de la prevalencia y las consecuencias sobre la salud de la población (2). El exceso de peso incrementa el riesgo de padecer enfermedades crónicas no transmisibles como diabetes tipo 2 e hipertensión, además de ocasionar un gran costo social ${ }^{(3)}$.

La prevención de la obesidad resulta compleja y exige un enfoque integral en el que participen todos los sectores gubernamentales y en el que las políticas de estado mejoren la salud de la población. La Organización Mundial de la Salud (OMS) ha elaborado un conjunto de recomendaciones para combatir la obesidad infantil. En dicho documento se hace un llamado para que los gobiernos tomen la iniciativa y reconozcan su responsabilidad para reducir el riesgo de obesidad en niños y adolescentes, en base a políticas de estado que generen intervenciones más allá del sector salud, algunos de los cuales analizaremos en el presente artículo ${ }^{(4)}$.

\section{EL PROBLEMA DE LA OBESIDAD}

La epidemia de obesidad podría afectar negativamente muchos de los beneficios de salud en la población mundial que han contribuido al aumento de longevidad en el mundo. Se ha estimado que, en el año 2014, 41 millones de niños menores de 5 años presentaban sobrepeso u obesidad ${ }^{(5)}$.

En el Perú, el estado nutricional de la población tiene la tendencia epidemiológica de mejora en sus condiciones socioeconómicas lo que favorece la disminución de

\footnotetext{
Servicio de Endocrinología y Metabolismo, Instituto Nacional de Salud del Niño. Lima, Perú

Facultad de Medicina Hipólito Unanue, Universidad Nacional Federico Villarreal. Lima, Perú

Médico endocrinólogo
}

Recibido: 10/01/2017 Aprobado: 25/01/2017 En línea: 22/03/2017

Citar como: Del Águila Villar CM. Obesidad en el niño: factores de riesgo y estrategias para su prevención en Perú. Rev Peru Med Exp Salud Publica. 2017;34(1):113-8. doi: 10.17843/rpmesp.2017.341.2773 
la desnutrición pero se observa un incremento de la obesidad. Actualmente, como consecuencia de ese cambio gradual, pueden coexistir la desnutrición crónica, el sobrepeso y la obesidad en los niños peruanos (6), siendo uno de los factores más importantes los cambios en la dieta y estilos de vida ocasionados por la urbanización y desarrollo económico ${ }^{(7)}$.

Según los reportes del observatorio de nutrición y estudio del sobrepeso y obesidad, en niños menores de cinco años, las cifras se han mantenido relativamente estables desde el año 2009 con un 6,8\% de sobrepeso y un $2,4 \%$ de obesidad al año 2014. En los últimos años, en niños de cinco a nueve años se presenta un incremento de la prevalencia de obesidad; pasando de $7,3 \%$ en el año 2008 a $14,8 \%$ en el periodo $2013-$ 2014. Del mismo modo, en adolescentes se observa un incremento de la prevalencia de $4,9 \%$ en el año 2007 a $7,5 \%$ en el periodo $2013-2014^{(8)}$.

Actualmente muchas enfermedades relacionadas con la obesidad consideradas frecuentemente en adultos se están reportando en niños con una tendencia cada vez mayor. Por ejemplo, la diabetes tipo 2, antes llamada diabetes del adulto, ahora aparece en niños, alrededor de los doce años ${ }^{(9)}$. Lo que indica que los niños tienen que hacer a esta enfermedad por un período más extenso, sin contar la implicancia económica. Se ha reportado que los gastos en atenciones médicas y en medicinas en adultos obesos fue de 36 y $77 \%$ más altos que en las personas no obesas, respectivamente. Wang et al. señalan que los gastos hospitalarios para el tratamiento de las enfermedades vinculadas con la obesidad infantil se incrementaron de $\$ 35$ millones a $\$ 127$ millones de 1979-1981 a 1997-1999 (10). Por lo tanto, la alta prevalencia de obesidad infantil y sus consecuencias obligan a prestar extrema atención a este problema de Salud Pública.

\section{FACTORES DE RIESGO}

El exceso de peso es un proceso gradual que suele iniciarse en la infancia y la adolescencia a partir de un desequilibrio entre la ingestión y gasto de energía, sumado a una predisposición genética expresada en una serie polimorfismos. Además, diversos cambios epigenéticos ocurren especialmente durante el embarazo, la infancia y la pubertad. Dichos cambios determinan una "memoria" de las experiencias tempranas, desencadenantes reversibles y susceptibilidad a la enfermedad en la vida adulta. De hecho, pueden ser heredadas a través de las divisiones de las células somáticas y potencialmente adquiridas a través de varias generaciones (epimutaciones) ${ }^{(11)}$.

Así, la obesidad durante la infancia está influenciada por factores genéticos, epigenéticos, conductuales y ambientales. Entre éstos, los factores conductuales y ambientales son más fácilmente modificables durante la infancia, por lo que estos son el foco de las intervenciones clínicas, de ahí la importancia de identificar factores de riesgo de obesidad en la población pediátrica. Estos factores de riesgo son los antecedentes familiares de obesidad, los malos hábitos de alimentación y el sedentarismo, entre otros.

En una cohorte de 1737 niños de 7 y 8 años realizada en Perú se ha reportado una prevalencia de sobrepeso de $19,2 \%$ y de obesidad de $8,6 \%$, los autores concluyen que pertenecer a un estrato socioeconómico alto, vivir en Lima, tener una madre con sobrepeso u obesidad; ser de sexo masculino y ser hijo único constituyen factores de riesgo importantes. Estos resultados indican una mayor predisposición al exceso de peso o a la obesidad entre los niños de hogares con mayores recursos económicos que entre los de hogares más pobres ${ }^{(12)}$.

Otro estudio realizado en 69,526 personas reporta una mayor prevalencia de sobrepeso y obesidad en adultos jóvenes $(62,3 \%)$ y una menor frecuencia en niños menores de 5 años $(8,2 \%)$ y además concluyen que el hecho de no ser pobre y vivir en un área urbana son determinantes sociales del exceso de peso en la población peruana. Del mismo modo, los resultados muestran una prevalencia de sobrepeso de $11,0 \%$ y obesidad de $3,3 \%$ en adolescentes peruanos ${ }^{(13)}$.

Un tema interesante es analizar el periodo de adiposidad de rebote. En general la masa corporal aumenta de manera rápida durante el primer año de vida y posteriormente disminuye hasta los seis años y después aumenta de nuevo. Los niños que desarrollan obesidad tiene un incremento prematuro de esa curva, el cual es llamado periodo de rebote de adiposidad, esto permite plantear estrategias de prevención para este grupo etario ${ }^{(14)}$. Dentro de los factores que condicionan este rebote de adiposidad, se considera, según diversos estudios, el exceso de proteínas al principio de la vida. Se ha observado que los niños que consumían más proteína tenían una rápida velocidad de crecimiento y por lo tanto mayor riesgo a obesidad ${ }^{(15,16)}$

Por otro lado, las dietas con alto contenido de grasas saturadas, carbohidratos de rápida absorción y poco consumo de grasas polinsaturadas y fibras, así como el incremento de sedentarismo, son algunas características de la transición epidemiológica nutricional, asimismo la mayor disponibilidad de alimentos de bajos costo ha permitido que la población pueda acceder a alimentos con alto contenido energético ${ }^{(17)}$

Además de la pobreza y el lugar de residencia existen factores sociales que podrían influenciar el incremento del sobrepeso y la obesidad, como son el nivel de 
educación. Al respecto, la educación está asociada inversamente al sobrepeso y a la obesidad y, por el contrario, el incremento del nivel socioeconómico está directamente relacionado con el sobrepeso y la obesidad por lo que estas condiciones ameritan mayor análisis ${ }^{(18)}$. Se ha reportado en niños peruanos, que a medida que se incrementa el nivel de pobreza también aumenta el consumo de carbohidratos y, por el contrario, disminuye el consumo de proteínas, minerales y vitaminas (19). Peña y Bacallao describen un obeso "pobre" y un obeso "rico" donde señala que el obeso pobre se encuentra sobrealimentado pero con deficiencias nutricionales lo cual agrega algunos problemas adicionales a este tipo de obesidad (20).

Asimismo, en países que tienen mayores ingresos económicos se describe mayor obesidad que los países con ingresos económicos más bajos. Otros factores como la disponibilidad y el acceso a los alimentos, propiciarían el consumo de alimentos de alto contenido energético, porque el consumidor selecciona los alimentos con un menor costo y no necesariamente por ser saludable; por tanto, es probable que se consuman alimentos con excesiva cantidad de carbohidratos, grasas y azúcares refinados ${ }^{(21)}$.

Las pruebas científicas disponibles apuntan a que lo más viable es gravar productos como las bebidas azucaradas, puesto que los datos indican que el impuesto repercute en el consumo. Puede que algunos países consideren la posibilidad de gravar otros alimentos no saludables, como los que tienen un alto contenido de grasas y azúcar. Para gravar los alimentos de alto contenido calórico y bajo valor nutricional sería necesario elaborar perfiles de nutrientes ${ }^{(22)}$; las predicciones sugieren que esto podría reducir el consumo.

Otro factor importante en el control del peso de niños es el núcleo familiar y su estilo de vida. Se ha reportado factores de riesgo modificables como la actividad física, la ingesta de desayuno diario y la educación de la persona que alimenta al niño como aspectos importantes a considerar en las políticas de salud pública, estos factores individuales y familiares deben de tomarse en cuenta cuando se diseñen estrategias de prevención contra la obesidad infantil (23).

Un estudio sobre la actividad física y hábitos alimentarios en niños obesos pertenecientes a un nivel socioeconómico bajo, reporto un bajo consumo de lácteos, verduras y frutas y un elevado consumo de bebidas azucaradas y alimentos con alta densidad energética. Más del 55\% de los niños no realizaban actividades físicas fuera del colegio. Los resultados indicaron deficiencias en las motivaciones y barreras para adquirir hábitos saludables de alimentación y de actividad física. A su vez, las madres mostraron poca motivación y baja predisposición para apoyar a sus hijos ${ }^{(24)}$.

En ese sentido, el estilo de crianza parece ser un factor que predispone a la obesidad en niños, un estudio señala que un $45 \%$ de niños con padres autoritarios tienen mayor facilidad de desarrollar obesidad en comparación a otros estilos de crianza, de ahí la importancia de rescatar algunas estrategias claves de puericultura implicados en una crianza responsable y alimentación saludable ${ }^{(25)}$.

\section{ESTRATEGIAS DE INTERVENCIÓN}

Los primeros años de vida son cruciales para establecer hábitos de alimentación saludable y de actividad física que reduzcan el riesgo de obesidad. La lactancia materna exclusiva durante los primeros seis meses de vida, seguida de la introducción de alimentos complementarios adecuados, juegan un rol importante en la reducción del riesgo de obesidad. Una adecuada alimentación complementaria junto con la prolongación de la lactancia materna puede reducir el riesgo de desnutrición y de acumulación de grasa corporal excesiva en los lactantes. Alentar la ingesta de diferentes alimentos sanos, en lugar de alimentos no saludables de alto contenido calórico y bajo valor nutricional y bebidas azucaradas, durante este periodo decisivo contribuye a un crecimiento y desarrollo óptimo. Los profesionales de la salud especialmente los pediatras pueden aprovechar el control del crecimiento y desarrollo del niño para hacer el seguimiento del índice de masa corporal (IMC) de los niños y dar a los padres asesoramiento adecuado para ayudar a prevenir que los niños padezcan sobrepeso y obesidad ${ }^{(26)}$.

Las directivas actuales sobre la alimentación complementaria (27) brindan orientación acerca del momento más adecuado para introducir esos alimentos, la alimentación perceptiva a las necesidades del niño, y la cantidad y el tipo de alimentos que se necesitan. Las actitudes de la familia ante la comida y sus percepciones sobre el peso corporal ideal son determinantes importantes de los comportamientos en torno a la alimentación complementaria y deben tenerse en cuenta.

Según datos científicos recientes, las experiencias sensoriales sobre los alimentos empiezan en el útero y continúan durante la lactancia materna, puesto que los sabores de los alimentos que consumen las madres se transmiten a sus lactantes. Este hecho y una alimentación complementaria adecuada pueden desempeñar una función importante en el establecimiento de las preferencias alimentarias y el control del apetito. Alentar a los niños al consumo de alimentos sanos mediante una exposición repetida ${ }^{(28)}$, que observen a los padres y a los 
miembros de la familia disfrutar de alimentos sanos y limitar la exposición de los niños a alimentos no saludables son estrategias que contribuyen a establecer buenos hábitos alimentarios en los niños y sus familias ${ }^{(29)}$.

Por otro lado, es necesario tener en cuenta que las mejores prácticas internacionales incluyen el incremento en la información que recibe el consumidor y la educación para cambiar las preferencias. Si las señales provenientes del mercado son confusas es difícil pensar que las compras realizadas por los consumidores serán las adecuadas y mucho menos las óptimas. Además, si el consumidor no está capacitado para hacer una interpretación o no entiende el mensaje que se envía, el resultado podría ser la compra de alimentos no saludables. Por lo tanto, es necesario realizar acciones que incrementen la información disponible respecto a las características, composición y las propiedades de los productos alimenticios, además de complementar con capacitación al consumidor, de ahí la importancia de las guías alimentarias, así como de normativas para una alimentación saludable ${ }^{(30,31)}$.

Existe evidencia que la promoción de alimentos no saludables y bebidas azucaradas está relacionada con la obesidad infantil ${ }^{(32)}$. La exposición a la promoción de alimentos no saludables sigue siendo un importante problema que exige tomar medidas para reducir la influencia negativa de esta exposición ${ }^{(33)}$.

Otros de los retos incluyen mejorar la identificación y el tratamiento para los pacientes con sobrepeso y obesidad en el primer nivel de atención. En ese sentido, la promoción de un estilo de vida activo y saludable es la herramienta más importante en la prevención y control de sobrepeso, obesidad y enfermedades crónicas. Ello implica el compromiso de intervención en todo niño que presente señales de alarma como el exceso de peso o antecedentes familiares que constituyan riesgo para obesidad, aun cuando esto no sea el motivo de la consulta.

Parte de un estilo de vida saludable es el fomento de la actividad física. Según reportes recientes, la actividad física desciende desde que el niño ingresa a la escuela ${ }^{(34,35)}$. En 2010 el $81 \%$ de los adolescentes entre 11 y 17 años no realizaban suficiente actividad física. Un $84 \%$ de las mujeres adolescentes y un $78 \%$ de hombres no llegaban los 60 minutos diarios de actividad física de intensidad moderada a intensa, según lo recomendado por la OMS ${ }^{(35)}$. La "modernidad" está ocasionando que realizar poco ejercicio sea visto como una norma social, siendo un factor importante en la epidemia de obesidad. La actividad física puede reducir el riesgo de diabetes, enfermedades cardiovasculares y el cáncer ${ }^{(36)}$.
La planificación y el diseño urbanísticos pueden agravar el problema y a la vez formar parte de la solución. Más espacios de recreo y carriles para desplazarse a pie y en bicicleta de forma segura ayudan realizar la actividad física necesaria cada día.

Los hábitos de práctica de ejercicio físico a lo largo de la vida pueden verse determinados en gran parte por las experiencias que se tienen de niño. Combatir la inseguridad ciudadana y propiciar comunidades seguras, que favorezcan la actividad física y en las que se permita y se impulse el transporte activo (desplazamiento a pie, en bicicleta, etc.) y la participación en actividades físicas y en un tipo de vida activo, redundará en beneficio de toda la sociedad. Es necesario prestar una atención especial para mejorar el acceso y participación en actividades físicas de los niños para prevenir el sobrepeso y la obesidad ${ }^{(37)}$.

Por todo ello, es urgente la implementación de intervenciones educativas que contribuyan a disminuir el exceso de peso de la población peruana, con énfasis en niños y adolescentes, a través de la promoción de los estilos de vida saludables en el núcleo familiar, así como el consumo de alimentos saludables, mayor actividad física con disminución de actividades sedentarias y menor número de horas de uso de medios audiovisuales y adecuadas horas de sueño en la población en general. Estas estrategias deben de ser aplicadas en los colegios donde los niños pasan la mayor parte de tiempo. Es importante la educación familiar en alimentación saludable, ingesta moderada de alimentos, propiciar el hábito del desayuno y evitar las comidas ricas en grasa por las noches, como estrategia para prevenir la obesidad en la infancia ${ }^{(38)}$

Nuestro país requiere planear, implementar estrategias y líneas de acción costo-efectivas dirigidas a la prevención y el control de la obesidad en el niño y adolescente. La experiencia global indica que la atención correcta de la obesidad y el sobrepeso requieren la formulación y coordinación de estrategias multisectoriales y eficientes que permitan potenciar los factores de protección hacia la salud, para modificar el comportamiento individual, familiar y comunitario.

En conclusión, la prevalencia del sobrepeso y la obesidad en niños y adolescentes constituyen un problema de salud pública emergente en el Perú, por lo que la identificación de los factores de riesgo debe formar parte de las estrategias de prevención para asegurar que la población infantil llegue a ser adulta sin enfermedades crónicas y con adecuada calidad de vida.

Fuente de financiamiento: este estudio fue autofinanciado

Conflicto de interés: el autor declara que no existe conflicto de interés en la publicación de este artículo. 


\section{REFERENCIAS BIBLIOGRÁFICAS}

1. Lake A, Townshend T. Obesogenic environments: exploring the built and food environments. J R Soc Promot Health. 2006;126(6):262-7.

2. Low S, Chew Chin M, DeurenbergYap M. Review on Epidemic of Obesity. Ann Acad Med Singapore. 2009;38(1):57-65.

3. Flegal KM, Graubard BI, Williamson DF, Gail MH. Cause-specific excess deaths associated with underweight, overweight, and obesity. JAMA. 2007;298(17):2028-37.

4. Organización Mundial de la Salud. Informe de la comisión para combatir la obesidad. Ginebra: OMS; 2016.

5. UNICEF, WHO, The World Bank Group. Joint child malnutrition estimates. Levels and trends [Internet]. Geneva: World Health Organization; 2015 [citado el 2 de diciembre de 2016]. Disponible en: http://www.who.int/ nutgrowthdb/estimates2014/en/

6. Mispireta ML, Rosas AM, Velásquez JE, Lescano AG, Lanata CF. Transición Nutricional en el Perú, 1991 - 2005. Rev Peru Med Exp Salud Publica. 2007;24(2):129-35.

7. Barria RM, Amigo H. Transición Nutricional: una revisión del perfil latinoamericano. Arch Latinoam Nutr. 2006;56(1):3-11.

8. Observatorio de Nutrición y Estudio del Sobrepeso y Obesidad [internet]. Lima: Instituto Nacional de Salud; c2017 [citado el 2 de diciembre de 2016]. Disponible en: http://www.observateperu.ins.gob.pe

9. Calagua M, Falen J, Del Águila C, Lu R, Rojas M. Características clínicas y bioquímicas de la diabetes mellitus tipo 2 (DMt2) en el Instituto Nacional de Salud del Niño. An Fac med. 2012;73(2):141-6.

10. Wang Y, Lim H. The global childhood obesity epidemic and the association between socio-economic status and childhood obesity. Int Rev Psychiatry. 2012;24(3):176-188 doi:10.3109/095 40261.2012.688195.

11. Manco M, Dallapiccola B. Genetics of pediatric obesity. Pediatrics. 2012;130(1):123-33. doi: 10.1542/ peds.2011-2717.

12. Preston E, Ariana E, Penny ME, Frost M, Plugge E. Prevalence of childhood overweight and obesity and associated factors in Peru. Rev Panam Salud Publica 2015;38(6):472-8.

13. Álvarez-Dongo D, Sánchez-Abanto J, Gómez-Guizado G, Tarqui-Mamani C. Sobrepeso y obesidad: prevalencia y determinantes sociales del exceso de peso en la población peruana (20092010). Rev Peru Med Exp Salud Publica. 2012; 29(3):303-13.

14. Rolland CMF, Deheeger M, Maill T, Bellisle F. Early adiposity rebound: causes and consequences for obesity in children and adults. Int J Obes (Lond) 2006;30(Suppl 4): S11-S17. doi: 10.1038/sj.ijo.0803514.

15. Michaelsen KF, Greer FR. Protein need early in life and long-term health. Am J Clin Nutr 2014; 99(3):718S-22S. doi: $10.3945 /$ ajen.113.072603

16. Hornell A, Lagstrom H, Lande B, Thorsdottir I. Protein intake from 0 to 18 years of age and its relation to health: a systematic literature review for the Nordic Nutrition Recommendations. Food Nutr Res 2013; 57:210-23. doi: 10.3402/fnr.v57i0.21083. Print 2013.

17. Orden AB, Torres MF, Luis MA, Cesani MF, Quintero FA, Oyhenart EE. Evaluación del estado nutricional en escolares de bajos recursos socioeconómicos en el contexto de la transición nutricional. Arch Argent Pediatr. 2005;103(3):205-11.

18. Fortich R, Gutiérrez J. Los determinantes de la obesidad en Colombia. Economía \& Región. 2011;5(2):155-82.

19. Rojas C, Calderón MDP, Taipe MDC, Bernui I, Ysla M, Riega V. Consumo de energía y nutrientes, características socioeconómicas, pobreza y área de residencia de niños peruanos de 12 a 35 meses de edad. Rev Peru Med Exp Salud Publica. 2004;21(2):98-106.

20. Peña M, Bacallao J. La Obesidad en la pobreza: un problema emergente en las Américas. En: Peña $M$, Bacallao J, editors. La obesidad en la pobreza: un nuevo reto para la Salud Pública. Washington: Organización Panamericana de la Salud; 2000. p. 3-11.

21. Ortiz-Moncada R, Alvarez-Dardet C, Miralles-Bueno JJ, Ruız-Cantero MT,
Dal Re-Saavedra MA, Villalba C, et al. Determinantes sociales de sobrepeso y obesidad en España 2006. Med Clin (Barc). 2011;137(15):678-84. doi: 10.1016/j.medcli.2010.12.025.

22. Powell LM, Chriqui JF, Khan T, Wada R, Chaloupka FJ. Assessing the potential effectiveness of food and beverage taxes and subsidies for improving public health: a systematic review of prices, demand and body weight outcomes. Obes Rev. 2013;14(2):110-28. doi: 10.1111/ obr.12002

23. Sánchez-Cruz JJ, de Ruiter I, JiménezMoleón JJ. Individual, family and environmental factors associated with pediatric excess weight in Spain: a cross-sectional study. BMC Pediatr 2014, 14:3-8. doi: 10.1186/14712431-14-3.

24. Olivares S, Bustos N, Moreno X, Lera $\mathrm{L}$, Cortez $\mathrm{S}$. Actitudes y prácticas sobre alimentación y actividad física en niños obesos y sus madres en Santiago, Chile. Rev chil Nutr. 2006;33(2):170-9.

25. Kakinami L, Barnett TA, Seguin L, Paradis G. Parenting style and obesity risk in children. Prev Med. 2015; 75:18-22. doi: 10.1016/j. ypmed.2015.03.005.

26. Horta BL, Loret de Mola C, Victora CG. Long-term consequences of breastfeeding on cholesterol, obesity, systolic blood pressure and type 2 diabetes: a systematic review and meta-analysis. Acta Paediatr. 2015;104(467):30-7. doi: 10.1111/ apa.13133.

27. Organización Panamericana de la Salud. Principios de orientación para la alimentación complementaria del niño amamantado. Washington: OPSOMS, 2003.

28. Mennella JA, Nicklaus S, Jagolino AL, Yourshaw LM. Variety is the spice of life: strategies for promoting fruit and vegetable acceptance during infancy. Physiol Behav. 2008;94(1):29-38. doi: 10.1016/j.physbeh.2007.11.014.

29. Liem DG, Mennella JA. Sweet and sour preferences during childhood: role of early experiences. Dev Psychobiol. 2002;41(4):388-95. doi: 10.1002/ dev.10067. 
30. Hawkes C, Smith TG, Jewell J, Wardle J, Hammond RA, Friel S, et al. Smart food policies for obesity prevention. Lancet. 2015;385(9985):2410 21 doi: $10.1016 /$ S01406736(14)61745-1.

31. Colchero MA, Popkin BM, Rivera JA, Ng SW. Beverage purchases from stores since the start of the Mexican sugar-sweetened beverage excise tax: a year out. BMJ. 2016;352:h6704. doi: 10.1136/bmj.h6704.

32. McGinnis JM, Gootman JA, Kraak VI. Food marketing to children and youth. Threat or opportunity? Washington: The National Academies Press; 2006.

33. World Health Organization A framework for implementing the set of recommendations on the marketing of foods and non-alcoholic beverages to children. Ginebra: Organización Mundial de la Salud, 2012.

34. Tremblay MS, Gray CE, Akinroye K, Harrington DM, Katzmarzyk PT, Lambert EV, et al. Physical activity of children: a global matrix of grades comparing 15 countries. J Phys Act Health. 2014;11:S113-25. doi: 10.1123/jpah.2014-0177.

35. OMS. Recomendaciones mundiales sobre actividad física para la salud. Ginebra: Organización Mundial de la Salud, 2010. Disponible en:

36. http://www.who.int/dietphysicalactivity/ factsheet_recommendations/es

37. World Cancer Research Fund; American Institute for Cancer Research. Food, nutrition, physical activity, and the prevention of cancer: a global perspective. Washington, D.C.:
World Cancer Research Fund; 2007.

38. LeBlanc AG, Spence JC, Carson V, Connor Gorber S, Dillman C, Janssen I, et al. Systematic review of sedentary behaviour and health indicators in the early years (aged 0-4 years). Appl Physiol Nutr Metab. 2012;37(4):75372. doi: 10.1139/h2012-063.

39. Huang JY, Qi SJ. Childhood obesity and food intake. World J Pediatr 2015;11(2):101-7. doi: 10.1007/ s12519-015-0018-2.

Correspondencia: Carlos Manuel Del Aguila Villar

Dirección: Hermanos Catari 240 - Dpto. 304 Maranga San Miguel. Lima, Perú Teléfono: (511) 999653731

Correo electrónico: caguilav@hotmail.com

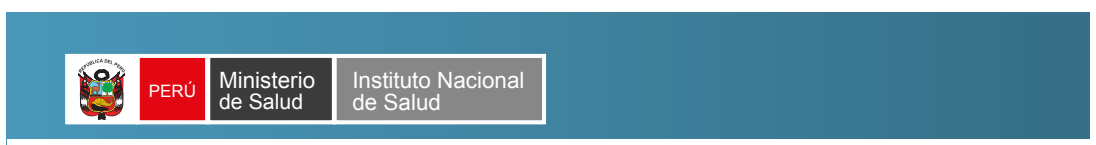

Inclusión social en salud: acercando el diagnóstico de dengue a las poblaciones afectadas

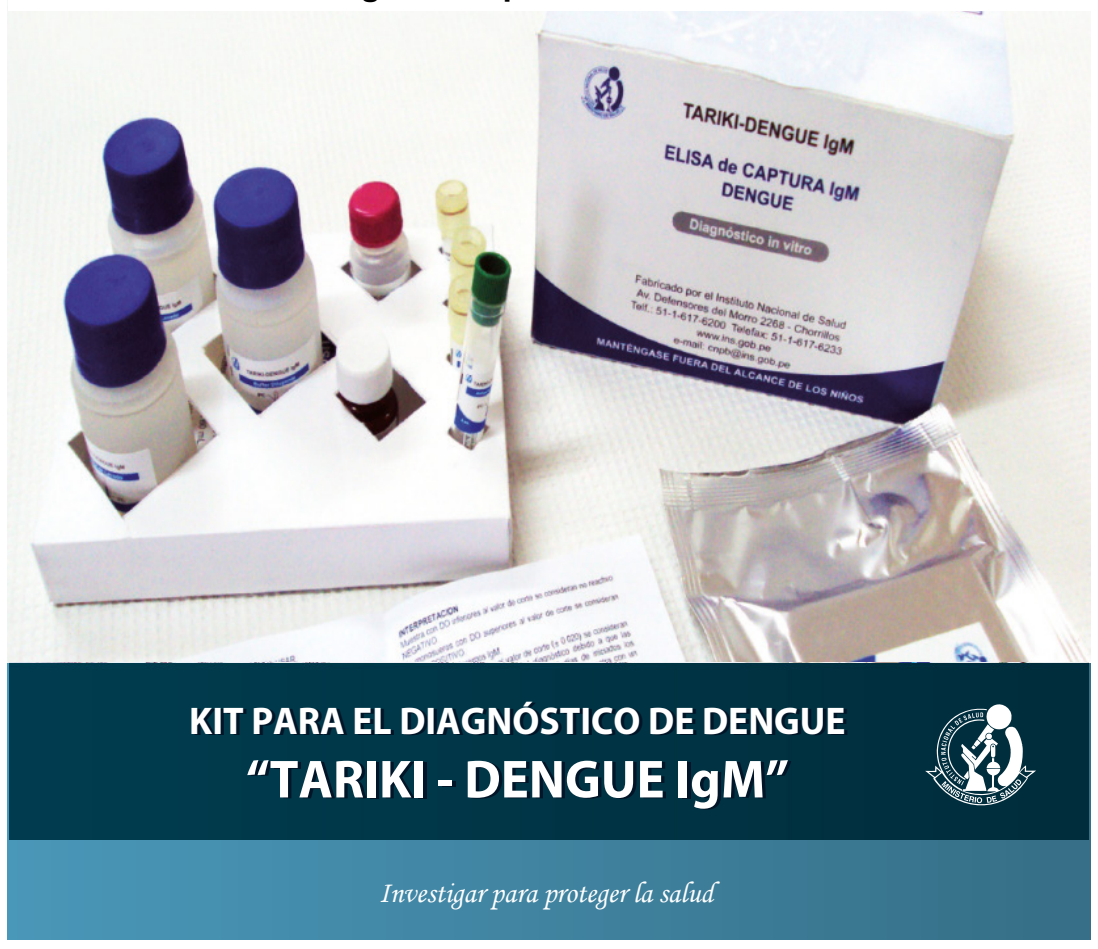

